Article

\title{
Breaking of Odd Chirality in Magnetoelectrodeposition of Copper Films on Micro-Electrodes
}

\author{
Iwao Mogi ${ }^{1, * \mathbb{C}}$, Ryoichi Aogaki ${ }^{2}$ and Kohki Takahashi ${ }^{1}$ \\ 1 Institute for Materials Research, Tohoku University, Katahira, Aoba-ku, Sendai 980-8577, Japan; \\ kohki@imr.tohoku.ac.jp \\ 2 Department of Product Design, Polytechnic University, Sumida, Tokyo 130-0026, Japan; ryoaochan@aol.com \\ * Correspondence: iwao.mogi.d4@tohoku.ac.jp
}

check for

updates

Citation: Mogi, I.; Aogaki,

R.; Takahashi, K. Breaking

of Odd Chirality in

Magnetoelectrodeposition of

Copper Films on Micro-Electrodes.

Magnetochemistry 2021, 7, 142.

https://doi.org/10.3390/

magnetochemistry7110142

Academic Editor: Catherine

P. Raptopoulou

Received: 17 September 2021

Accepted: 20 October 2021

Published: 27 October 2021

Publisher's Note: MDPI stays neutral with regard to jurisdictional claims in published maps and institutional affiliations.

Copyright: (c) 2021 by the authors. Licensee MDPI, Basel, Switzerland. This article is an open access article distributed under the terms and conditions of the Creative Commons Attribution (CC BY) license (https:/ / creativecommons.org/licenses/by/ $4.0 /)$.

\begin{abstract}
The surface chirality was investigated in magnetoelectrodeposition (MED) of copper films on micro-disc electrodes with the diameters of 100 and $25 \mu \mathrm{m}$. The MED was conducted in the magnetic fields of 1-5 T, which were parallel or antiparallel to the ionic currents. In the case of $100 \mu$ m-electrodes, the MED films prepared in 2 and $3 \mathrm{~T}$ exhibited odd chirality for the magnetic field polarity, as expected in the magnetohydrodynamic (MHD) vortex model. However, the films prepared in the higher fields of 4 and $5 \mathrm{~T}$ exhibited breaking of odd chirality. In the case of the $25 \mu \mathrm{m}$-electrode, the broken odd chirality was observed in 2 and $3 \mathrm{~T}$. These results indicate that the strong vertical MHD flows induce the breaking of odd chirality. The mapping of chiral symmetry on the axes of the magnetic field and electrode diameter demonstrate that the odd chirality could be easily broken by the fluctuation of micro-MHD vortices.
\end{abstract}

Keywords: surface chirality; magnetoelectrodeposition; alanine; micro-MHD vortex; micro-electrode; chiral symmetry breaking

\section{Introduction}

Magnetoelectrodeposition (MED), which represents electrodeposition under magnetic fields [1], can induce surface chirality on copper and silver thin films [2,3]. When the MED films were used as electrodes, they exhibited chiral responses for the oxidation reactions of enantiomers of amino acids, tartaric acid, and glucose [4-6]. The chiral surface formation in the MED processes is related to the magnetohydrodynamic (MHD) flows and vortices caused by the Lorentz force $[7,8]$.

The chiral surfaces on MED films can be formed in the magnetic fields perpendicular to the working electrode. The Lorentz force brings about macroscopic and microscopic flows around the electrode [7,8], as shown in Figure 1a. The vertical MHD flow is induced around the electrode edge, where the currents $\boldsymbol{i}$ are not parallel to the magnetic field $\boldsymbol{B}$. On the other hands, the micro-MHD vortices are excited around the local bumps and pits, which are caused by non-equilibrium fluctuation on the deposit surfaces. There exists a number of screw dislocations on electrodeposited copper films [9]. The micro-MHD vortices could contribute to the chiral site formation on the screw dislocations [8]. The vortices form the self-organized state, where the clockwise and anticlockwise vortices adjoin each other. The self-organized state leads to network structures on the surface morphology of the MED films [5]. Such symmetrical self-organized state is disturbed by the influence of vertical MHD flows. The cyclonic vortices are stable and they can contribute to the chiral site formation, whereas anticyclonic ones become unstable [10,11]. The direction of vertical MHD flow depends on the magnetic field polarity, thus the chiral sign of MED film becomes opposite for the reverse of magnetic field polarity. This behavior represents the odd chirality for the magnetic field polarity [12].

The odd chirality was clearly observed in the MED of copper films at $5 \mathrm{~T}$ on the usual $\mathrm{mm}$-size electrode $[5,13]$. This fact indicates the formation of rigid self-organized state 
of micro-MHD vortices under the vertical MHD flows as depicted in Figure 1a. On the other hand, the same chiral sign was observed in both magnetic field polarities under several MED conditions: specific adsorption of chloride ions [13], low magnetic fields [14], and cell rotation [15]. For example, the copper films prepared by the MED with chloride additives showed only L-activity in both magnetic field polarities at $5 \mathrm{~T}$ [13]: The MED films prepared at $2.5 \mathrm{~T}$ showed L-activity in both polarities. Such MED conditions cause the fluctuation of micro-MHD vortices, hence the fluctuation would be crucial for the broken odd chirality.

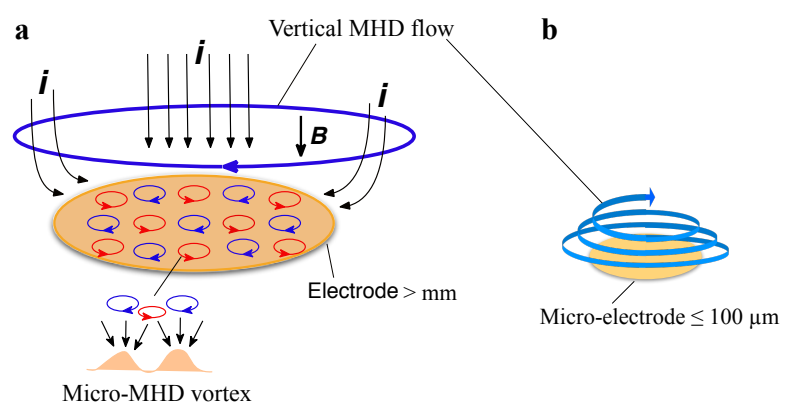

Figure 1. (a): Schematic of self-organized state of micro-MHD vortices under the vertical MHD flow in a MED process with a usual-size $(\mathrm{mm})$ electrode. $(\mathbf{b})$ : Vertical MHD flows on a micro-electrode.

The breaking of odd chirality is of great interest in connection with the origin of homochirality of amino acids in biochemical systems. We have explored the MED conditions to induce the broken odd chirality in copper films as mentioned above. Here our attention is focused on the influence of vertical MHD flows on the micro-MHD vortices. When the electrode downsizes to micrometer scales, the vertical MHD flows envelop the whole area of electrode and strongly affect the micro-MHD vortices as shown in Figure 1b, leading to the fluctuation of micro-MHD vortices. In this study, we investigate the chiral behaviors of MED copper films on the micro-disc-electrode with diameter of 100 and $25 \mu \mathrm{m}$, and here we show that the appearance of broken odd chirality depends on the magnetic field and the electrode diameter.

\section{Results and Discussion}

\subsection{Chiral Behaviors of MED Films on a $100 \mu \mathrm{m}$-Electrode}

The MED of copper films was conducted on a micro-disc electrode with $100 \mu \mathrm{m}$ diameter in the magnetic fields of $1-5 \mathrm{~T}$, which were parallel $(+B)$ or antiparallel $(-B)$ to the ionic currents. The film prepared in a magnetic field of $+1 \mathrm{~T}$, for example, is termed the +1 T-film. To estimate the surface chirality of MED films, the films were employed as electrodes, and the voltammograms of alanine enantiomers were measured on the MED film electrodes in a $0.1 \mathrm{M} \mathrm{NaOH}$ aqueous solution. Figure 2a shows voltammograms of L- and D-alanines on the +2 T-film electrode prepared at a deposition current of $19 \mathrm{~mA}$ $\mathrm{cm}^{-2}$. Alanine molecules are oxidized around $0.7 \mathrm{~V}$ on a copper electrode [16], resulting in the current peaks in Figure 2a. The surface chirality of MED films can be reflected in the peak currents of enantiomers. The peak current of D-alanine is greater than that of L-alanine. This fact represents that the $+2 \mathrm{~T}$-film electrode has D-activity. On the contrary, the $-2 \mathrm{~T}$-film electrode exhibits L-activity as shown in Figure 2b.

The chirality in voltammograms was evaluated by an enantiomeric excess (ee) ratio defined as

$$
e e=\left(i_{\mathrm{p}}{ }^{\mathrm{L}}-i_{\mathrm{p}} \mathrm{D}\right) /\left(i_{\mathrm{p}}{ }^{\mathrm{L}}+i_{\mathrm{p}} \mathrm{D}\right)
$$

where $i_{\mathrm{p}} \mathrm{L}$ and $i_{\mathrm{p}} \mathrm{D}$ represent the peak currents of L- and D-alanines, respectively. The positive and negative signs of $e e$ ratios stand for L- and D-activity, respectively. As reported in our previous papers [13], the surface chirality depends on the deposition current, thus the MED films were prepared at various deposition currents. Figure $3 a, b$ show the deposition current dependence of $e e$ ratios (ee ratio profile) for the $+2 \mathrm{~T}$-film and $-2 \mathrm{~T}$-film electrodes, 
respectively. The $+2 \mathrm{~T}$-film shows D-activity in the current region around $19 \mathrm{~mA} \mathrm{~cm}{ }^{-2}$, on the contrary, the $-2 \mathrm{~T}$-film shows the L-activity at the same current region. This result can be described using the following diagrams:

$$
e e(B) \approx-e e(-B) .
$$

Such a relation represents the odd chirality for the magnetic field polarity. The odd chirality is easily understood by the self-organized MHD model in Figure 1a, as described above.
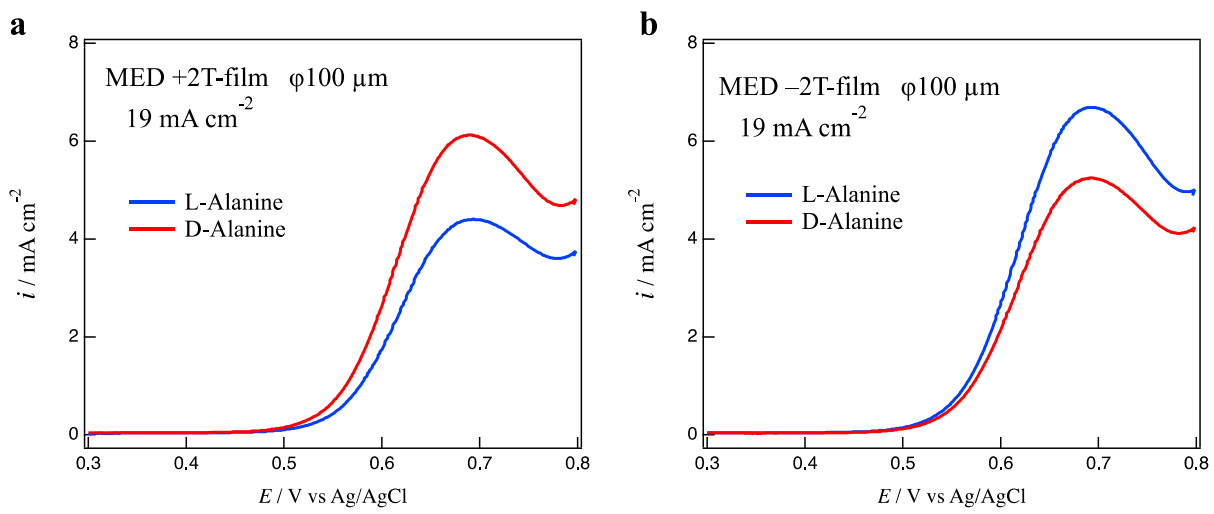

Figure 2. Voltammograms of alanine enantiomers on the 2 T-film electrodes prepared on a $100 \mu \mathrm{m}$ electrode with deposition current of $19 \mathrm{~mA} \mathrm{~cm}^{-2}$; (a): the +2 T-film electrode, (b): the $-2 \mathrm{~T}$-film electrode.
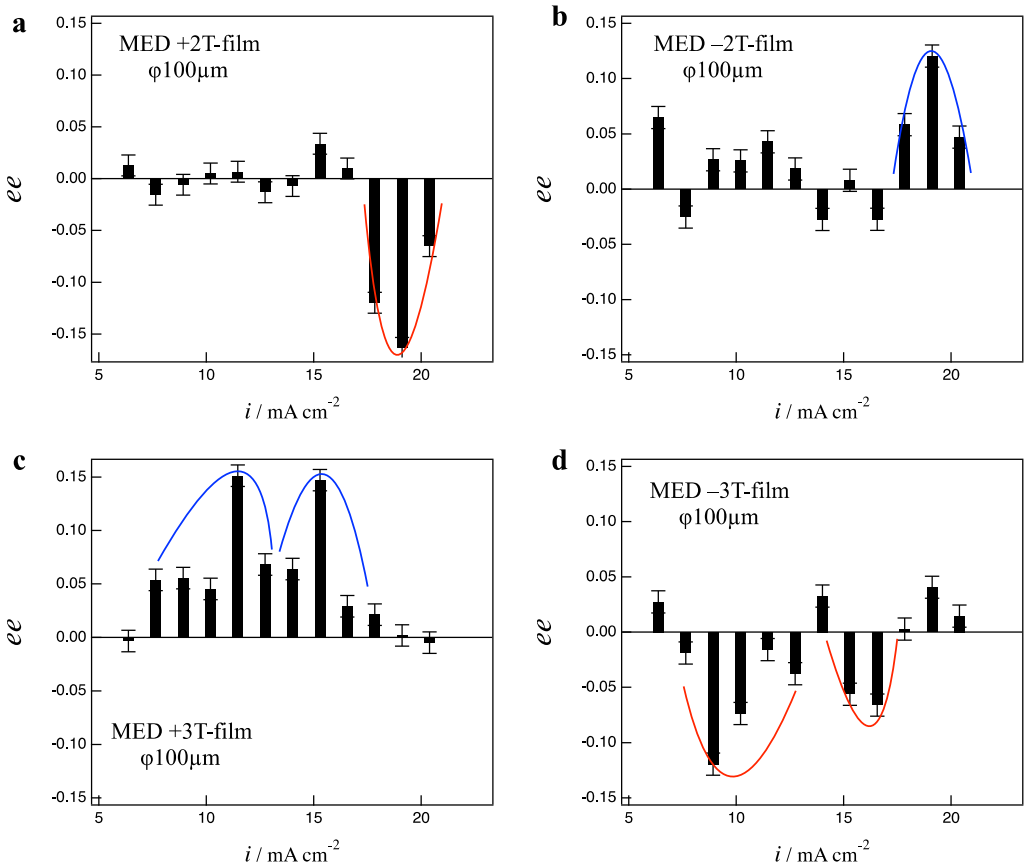

Figure 3. The ee ratios vs deposition currents on a $100 \mu$ m-electrode. (a); +2 T-film, (b); -2 T-film, (c); +3 T-film and (d); -3 T-film. Blue and red curves are a guide for eyes.

Similar odd chirality can be seen in the $3 \mathrm{~T}$-films. Figure $3 \mathrm{c}$,d show the ee ratio profiles for the $+3 \mathrm{~T}$ - and -3 T-film electrodes, respectively. The $+3 \mathrm{~T}$-films exhibit L-activity in the wide current region of 7-17 mA cm${ }^{-2}$, and the $-3 \mathrm{~T}$-films exhibit D-activity in the same region. The results in Figure 3 indicate that the self-organized MHD state is formed in the MED at 2 and $3 \mathrm{~T}$ even on the $100 \mu \mathrm{m}$-electrodes. 
When the MED is conducted in the higher magnetic fields, the influence of vertical magnetic field is expected to be more substantial. Figure $4 \mathrm{a}, \mathrm{b}$ show the $e e$ ratio profiles for the $+4 \mathrm{~T}$ - and $-4 \mathrm{~T}$-film electrodes, respectively. The $+4 \mathrm{~T}$-film exhibits L-activity in the current region of 6-15 $\mathrm{mA} \mathrm{cm}^{-2}$, and the -4 T-film also exhibits L-activity in the same current region. Such behaviors represent the clear breaking of odd chirality, rather even chirality for the magnetic field polarity.
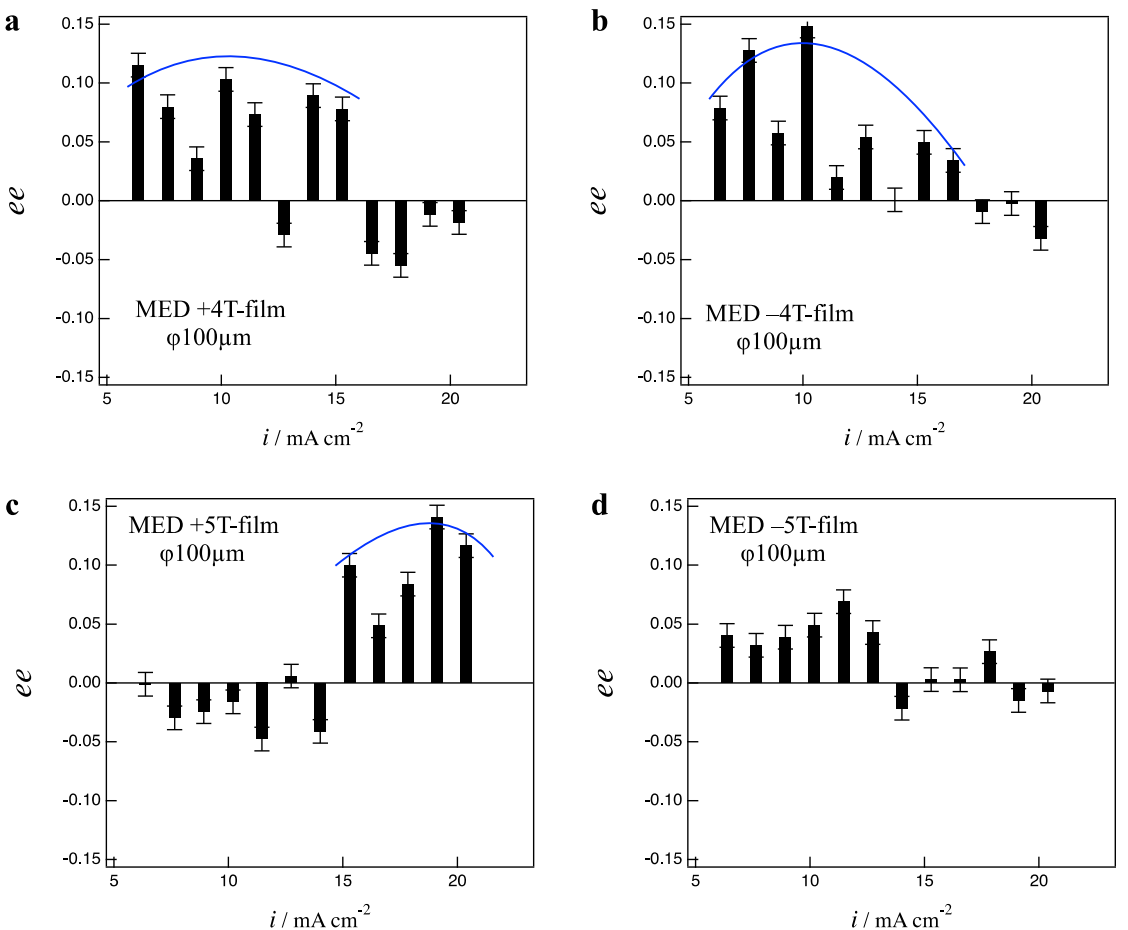

Figure 4. The ee ratios vs deposition currents on a $100 \mu$ m-electrode. (a); +4 T-film, (b); -4 T-film, (c); +5 T-film and (d); -5 T-film. Blue curves are a guide for eyes.

Figure $4 \mathrm{c}$, d show the ee ratio profiles for the $+5 \mathrm{~T}$ - and $-5 \mathrm{~T}$-film electrodes, respectively. The +5 T-film exhibits L-activity in the current region of $15-21 \mathrm{~mA} \mathrm{~cm}^{-2}$, on the other hand, the $-5 \mathrm{~T}$-film exhibits achirality in the same current region. These facts also represent the breaking of odd chirality. The results in Figure 4 indicate that the strong influence of vertical MHD flows causes the fluctuation of micro-MHD vortices, leading to the breaking of odd chirality. When the influence of vertical MHD flows is strong enough, the microMHD vortices have random fluctuation, leading to the disappearance of surface chirality as observed in the $-5 \mathrm{~T}$-film (Figure $4 \mathrm{~d}$ ). When the influence is not so strong, the microMHD vortices have "ordered fluctuation". Such ordered fluctuation could lead to the even chirality as observed in 4 T-films.

The ordered and random fluctuations of micro-MHD vortices were proposed in our previous paper [14], where the effects of low magnetic fields on the surface chirality were studied with and without the specific adsorption of chloride ions. The low magnetic fields induce ordered fluctuation, leading to the even chirality for the magnetic field polarity. The even chirality is forbidden symmetry on the basis of the simple MHD model. A number of MED results [13-15] suggest that the ordered fluctuation is crucial for the forbidden symmetry. On the other hand, the superimposed effects of low magnetic fields and specific adsorption induce random fluctuation, leading to the disappearance of surface chirality.

When the MED was conducted in the magnetic field lower than $2 \mathrm{~T}$, the effects of low magnetic fields can be expected even in the micro-electrodes. Figure $5 \mathrm{a}, \mathrm{b}$ show the ee ratio profiles for the $+1 \mathrm{~T}$ - and $-1 \mathrm{~T}$-film electrodes, respectively. In the low current region of 6-10 $\mathrm{mA} \mathrm{cm}^{-2}$, the $+1 \mathrm{~T}$-film shows L-activity, and the $-1 \mathrm{~T}$-film shows Dactivity, representing the odd chirality. On the contrary, in the middle current region of 
12-16 $\mathrm{mA} \mathrm{cm}^{-2}$, both $+1 \mathrm{~T}$ - and $-1 \mathrm{~T}$-films show D-activity, representing the even chirality. The ee ratio profiles of $1 \mathrm{~T}$-films exhibit the coexistence of the odd and even chirality. In the low magnetic field of $1 \mathrm{~T}$, the vertical MHD flows have tiny influence, instead, the low magnetic field causes the fluctuation of micro-MHD vortices, leading to the breaking of odd chirality.
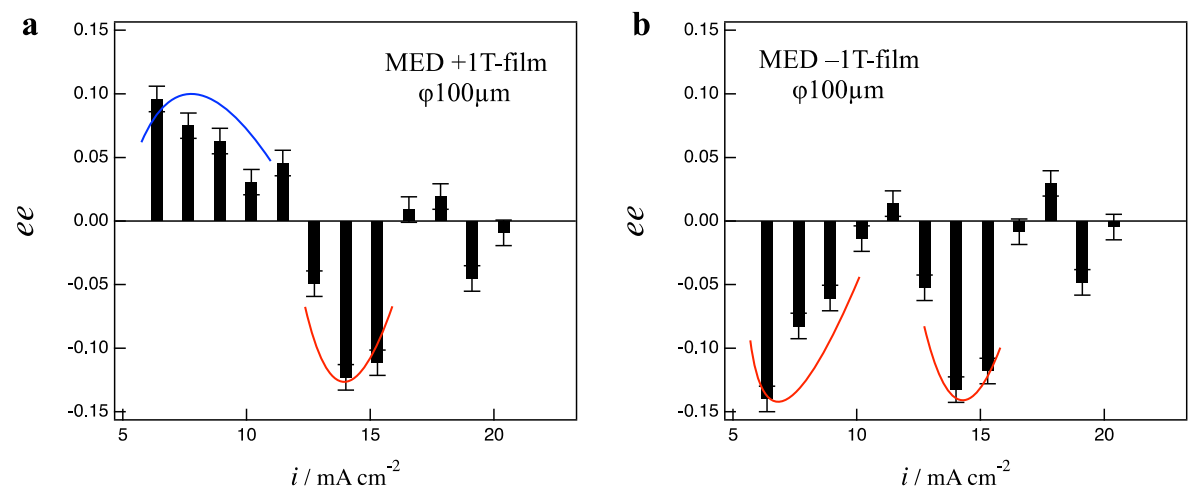

Figure 5. The $e e$ ratios vs deposition currents on a $100 \mu \mathrm{m}$-electrode. (a); +1 T-film, (b); -1 T-film. Blue and red curves are a guide for eyes.

\subsection{Chiral Behaviors of MED Films on a $25 \mu m$-Electrode}

The influence of vertical MHD flows on the micro-MHD vortices depends on the electrode diameter. As the electrode is smaller, the influence would spread over the whole electrode surface, and it becomes more remarkable. To examine the effects of smaller electrode, the MED of copper films was conducted on a micro-disc electrode with $25 \mu \mathrm{m}$ diameter. Figure $6 \mathrm{a}, \mathrm{b}$ show the $e e$ ratio profiles for the $+2 \mathrm{~T}$ - and $-2 \mathrm{~T}$-film electrodes, respectively. Both $+2 \mathrm{~T}$ - and $-2 \mathrm{~T}$-films show D-activity in the wide current region of 6-30 $\mathrm{mA} \mathrm{cm}^{-2}$, representing even chirality. While the $2 \mathrm{~T}$-films exhibit clear odd chirality on the $100 \mu \mathrm{m}$-electrodes (Figure 4), they exhibit the breaking of odd chirality on the $25 \mu \mathrm{m}$-electrodes. This indicates that the fluctuation of micro-MHD vortices is ascribed to the vertical MHD flows.

The breaking of odd chirality can be seen in the 3 T-film as shown in Figure $6 c, d$, where the -3 T-film show clear D-activity around 10 and $30 \mathrm{~mA} \mathrm{~cm}^{-2}$, on the other hand, the $+3 \mathrm{~T}$-film show almost achirality except tiny D-activity around 10 and $30-40 \mathrm{~mA} \mathrm{~cm}^{-2}$. As reported in our previous paper [17], both $+5 \mathrm{~T}$ - and $-5 \mathrm{~T}$-films showed achirality on the $25 \mu \mathrm{m}$-electrodes. The random fluctuation of micro-MHD vortices is dominant in the MED processes of the $+3 \mathrm{~T}$ - and $5 \mathrm{~T}$-films.

When the MED was conducted in the lower magnetic fields, the influence of vertical MHD flows could be suppressed even in the $25 \mu \mathrm{m}$-electrodes. Figure $7 \mathrm{a}, \mathrm{b}$ show the $e e$ ratio profiles for the $+1 \mathrm{~T}$ - and $-1 \mathrm{~T}$-film electrodes, respectively. The $+1 \mathrm{~T}$-film shows D-activity in the whole current region, in contrast, the $-1 \mathrm{~T}$-film shows L-activity, representing the clear odd chirality. While the $1 \mathrm{~T}$-films partially exhibits the breaking of odd chirality on the $100 \mu$ m-electrodes (Figure 5), they exhibit only odd chirality. This result suggests that the fluctuation effects arising from low magnetic fields could be suppressed in the smaller-size electrode. 
a

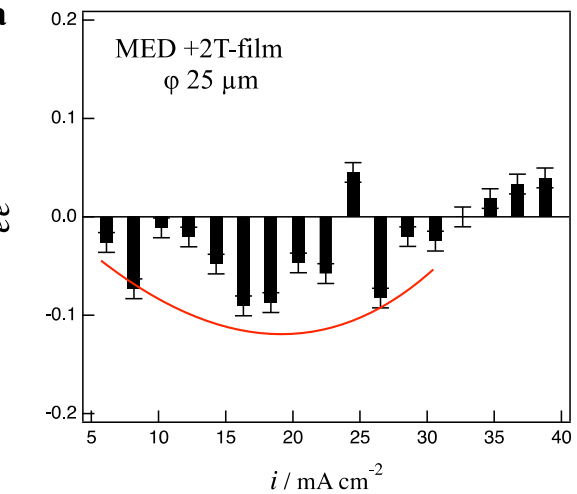

c

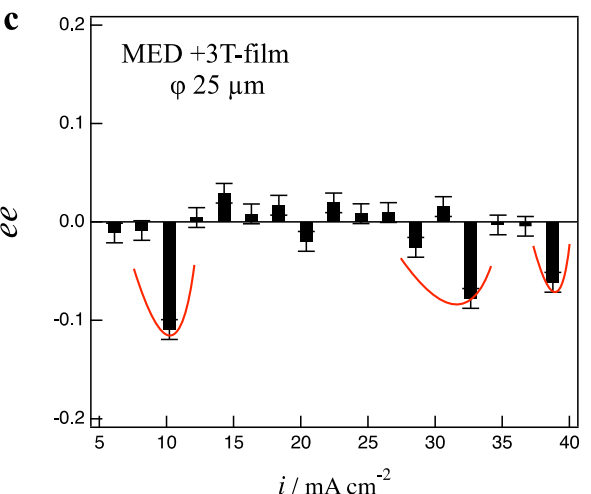

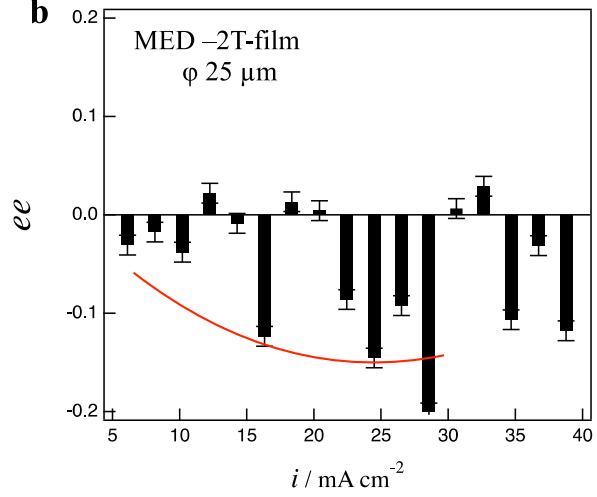

d

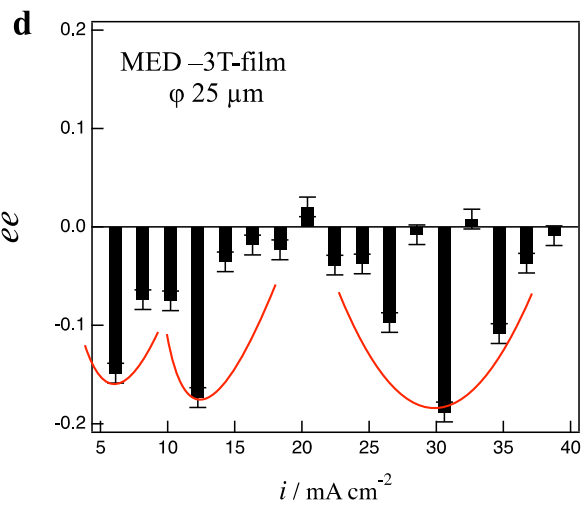

Figure 6. The ee ratios vs deposition currents on a $25 \mu$ m-electrode. (a); +2 T-film, (b); -2 T-film, (c); +3 T-film and (d); -3 T-film. Red curves are a guide for eyes.
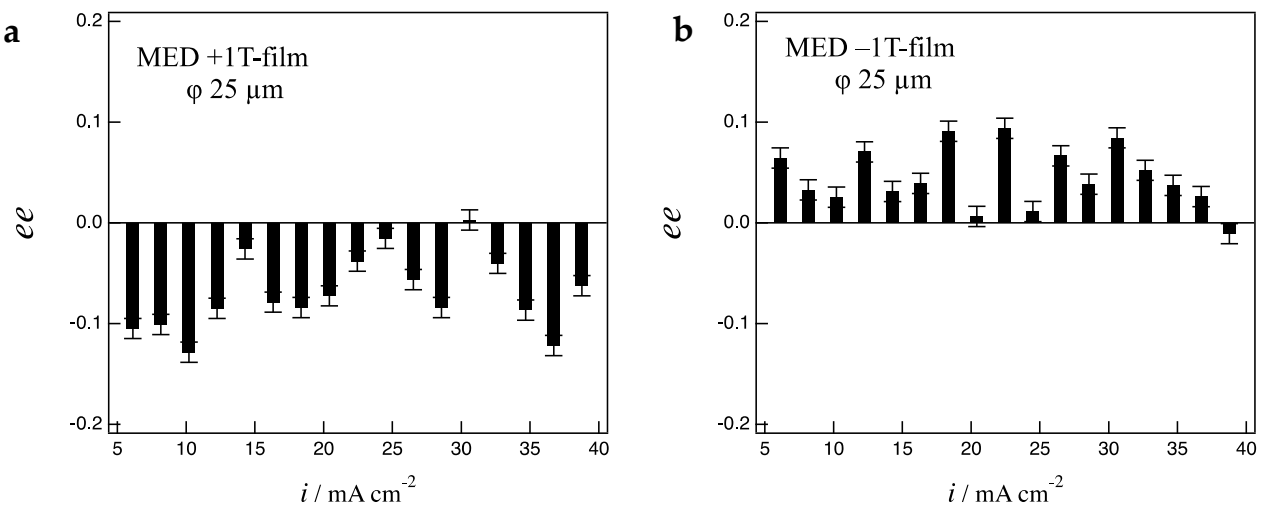

Figure 7. The ee ratios vs deposition currents on a $25 \mu$ m-electrode. (a); +1 T-film, (b); -1 T-film.

\subsection{Mapping of Chiral Symmetry}

We have shown the chiral symmetry behaviors of MED films in the magnetic fields of $1-5 \mathrm{~T}$ on the $100 \mu \mathrm{m}$ - and $25 \mu \mathrm{m}$-electrodes. Figure 8 shows the mapping of chiral symmetry on the axes of magnetic field vs. electrode diameter, including the results of $3 \mathrm{~mm}$-electrodes in our previous paper [14]. The chiral symmetry is classified into three types: odd chirality $(\mathrm{O})$ for the magnetic field polarity, breaking of odd chirality $(\mathrm{B})$ and achirality (A).

The surface chirality on copper MED films was found in the 5 T-film on the $3 \mathrm{~mm}$ electrode for the first time $[13,14]$. As the electrode size decreases, the influence of vertical MHD flows causes the ordered fluctuation of micro-MHD vortices, leading to the breaking of odd chirality (red-B) on the $100 \mu$ m-electrode. More drastic influence of vertical MHD flows causes the random fluctuation on the $25 \mu \mathrm{m}$-electrode, leading to the achirality. On the other hand, as the magnetic field decreases on the $3 \mathrm{~mm}$-electrode, the ordered 
fluctuation of micro-MHD vortices emerges at 2-2.5 T, leading to the breaking of odd chirality (pink-B). The self-organized state of micro-MHD vortices cannot well develop in the lower magnetic field of $1 \mathrm{~T}$, thereby the MED films show achirality.

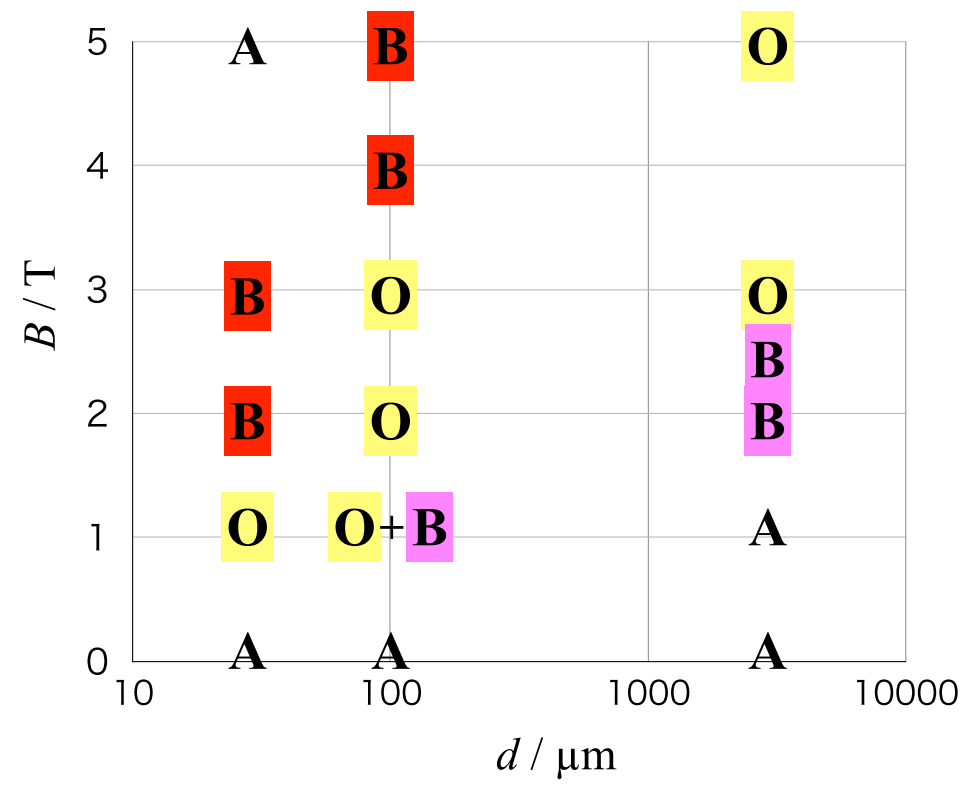

Figure 8. Mapping of chiral symmetry on the axes of magnetic field vs. electrode diameter $d$ (logarithmic scales). O: odd chirality; B: breaking of odd chirality; A: achirality.

The odd chirality appears at the lower magnetic fields of 1-3 $\mathrm{T}$ on the $100 \mu \mathrm{m}$ electrodes and at $1 \mathrm{~T}$ on the $25 \mu \mathrm{m}$-electrodes. The low magnetic fields reduce the influence of vertical MHD flows, thereby the self-organized state of micro-MHD vortices survives on the micro-electrodes. The breaking of odd chirality at the $1 \mathrm{~T}$-film on the $100 \mu \mathrm{m}$-electrodes implies the fluctuation effect induced by the low magnetic fields.

Figure 8 shows that the chiral surfaces are formed in the wide areas of magnetic field and electrode diameter (the types $\mathrm{O}$ and $\mathrm{B}$ ). It is remarkable that the chiral surface can be formed without chiral agents. There are two types of breaking of odd chirality: red-B and pink-B. The red-B is caused by the vertical MHD flows, and the pink-B is caused by the low magnetic fields. The area of type $\mathrm{O}$ is surrounded by those of type $\mathrm{B}$, and the achirality is in the outer areas. Hence, the odd chirality exists in the confined areas of magnetic field and electrode diameter. This fact implies that the odd chirality could be easily broken by the fluctuation of micro-MHD vortices.

\section{Materials and Methods}

The electrolytic cell in MED experiments consists of three electrodes: a polycrystalline platinum micro-disc working electrode with diameters of 100 and $25 \mu \mathrm{m}$ (ALS Co. Ltd., Tokyo, Japan), a copper plate counter electrode, and a $\mathrm{Ag}|\mathrm{AgCl}| 3 \mathrm{M}\left(\mathrm{M}=\mathrm{mol} \mathrm{dm}^{-3}\right)$ $\mathrm{NaCl}$ reference electrode. The copper films were electrodeposited galvanostatically on the working electrode at various constant currents of $5-40 \mathrm{~mA} \mathrm{~cm}^{-2}$ in a $50 \mathrm{mM} \mathrm{CuSO}_{4}$ $+0.5 \mathrm{M} \mathrm{H}_{2} \mathrm{SO}_{4}$ aqueous solution. The film thickness was approximately $150 \mathrm{~nm}$ at the passing charge of $0.4 \mathrm{C} \mathrm{cm}^{-2}$.

In the MED processes, the electrolytic cell was put on a cell holder at the bore center in a cryocooled solenoidal superconducting magnet (Sumitomo Heavy Industries Ltd., Tokyo, Japan). This magnet can produce magnetic fields of up to $5 \mathrm{~T}$ in a $220 \mathrm{~mm}$ roomtemperature bore. An applied magnetic field was parallel $(+B)$ or antiparallel $(-B)$ to the ionic current and perpendicular to the working electrode surface. The schematic of MED configuration was reported in our previous papers [5]. The temperature within the magnet bore was adjusted to $25^{\circ} \mathrm{C}$ by circulating thermo-controlled water. 
The chiral behaviors of MED films were examined by the voltammetric measurements of alanine enantiomers on the MED film electrodes. The films underwent the pre-treatment of a potential sweep $(-0.3-0.3 \mathrm{~V})$ in a $0.1 \mathrm{M} \mathrm{NaOH}$ aqueous solution [5]. The voltammograms of $20 \mathrm{mM}$ L- or D-alanine were measured on the MED film electrodes in a $0.1 \mathrm{M}$ $\mathrm{NaOH}$ aqueous solution with a linear potential sweep rate of $10 \mathrm{mV} \mathrm{s}^{-1}$ in the absence of a magnetic field.

\section{Conclusions}

We have shown the ee ratio profiles of copper MED films prepared in various conditions at $1-5 \mathrm{~T}$ on the $100 \mu \mathrm{m}$ - and $25 \mu \mathrm{m}$-electrodes and found that the odd chirality for the magnetic field polarity can be broken by the considerable influence of vertical MHD flows on the micro-MHD vortices. The mapping of chiral symmetry on the axes of magnetic field and electrode diameter exhibits that the odd chirality can exist in the confined area surrounded by those of broken odd chirality. This indicates that the chiral symmetry on the MED films could be easily broken by the fluctuation of micro-MHD vortices. These results would lead to significant hints for the origin of homochirality in biomolecules, taking account of the catalytic roles of chiral surfaces of minerals in the molecular evolution on the early earth [18].

Author Contributions: I.M. and R.A. conceived and designed the concept and experiments; I.M. and K.T. conducted the experiments; K.T. contributed to superconducting magnet tools; I.M. wrote the paper. All authors have read and agreed to the published version of the manuscript.

Funding: This research was funded by JSPS KAKENHI Grant-in-Aid for Scientific Research (C) No. 19K05230.

Institutional Review Board Statement: Not applicable.

Informed Consent Statement: Not applicable.

Data Availability Statement: Data presented in this study is available on request from the corresponding author.

Acknowledgments: The authors thank the staff members S.A. and H.N. of High Field Laboratory for Superconducting Materials of IMR Tohoku University for the use of the cryocooled superconducting magnet.

Conflicts of Interest: The authors declare no conflict of interest.

\section{References}

1. Fahidy, T.Z. Magnetoelectrolysis. J. Appl. Electrochem. 1983, 13, 553-563. [CrossRef]

2. Mogi, I.; Morimoto, R.; Aogaki, R. Surface Chirality Effects Induced by Magnetic Fields. Curr. Opin. Electrochem. $2018,7,1-6$. [CrossRef]

3. Kumar, A.; Mondal, P.C.; Fontanesi, C. Chiral Magneto-Electrochemistry. Magnetochemistry 2018, 4, 36. [CrossRef]

4. Mogi, I.; Watanabe, K. Chiral Electrode Behavior of Magneto-Electrodeposited Silver Films. ISIJ Int. 2007, 47, 585-587. [CrossRef]

5. Mogi, I.; Watanabe, K. Chiral Recognition of Amino Acids by Magnetoelectrodeposited Cu Film Electrodes. Int. J. Electrochem. 2011, 239637. [CrossRef]

6. Mogi, I.; Watanabe, K. Enantioselective Recognition of Tartaric Acid on Magnetoelectrodeposited Copper Film Electrodes. Chem. Lett. 2012, 41, 1439-1441. [CrossRef]

7. Aogaki, R. Micro-MHD Effect on Electrodeposition in Vertical Magnetic Field. Magnetohydrodynamics 2003, 4, 453-460.

8. Aogaki, R.; Morimoto, R. Nonequilibrium Fluctuations in Micro-MHD Effects on Electrodeposition. In Heat and Mass Transfer: Modeling and Simulation; Hossain, M., Ed.; InTech: London, UK, 2011; pp. 189-216.

9. Yanson, Y.I.; Rost, M.J. Structural Accelerating Effect of Chloride on Copper Electrodeposition. Angew. Chem. Int. Ed. 2013, 52, 2454-2458. [CrossRef] [PubMed]

10. Sommeria, J.; Meyers, S.D.; Swinney, H.L. Laboratory Simulation of Jupiter's Great Red Spot. Nature 1988, 331, 689-693. [CrossRef]

11. Marcus, S.M. Numerical Simulation of Jupiter's Great Red Spot. Nature 1988, 331, 693-696. [CrossRef]

12. Rikken, G.L.; Folling, J.; Wyder, P. Electrical Magnetochiral Anisotropy. Phys. Rev. Lett. 2001, 87, 236602. [CrossRef] [PubMed]

13. Mogi, I.; Aogaki, R.; Watanabe, K. Tailoring of Surface Chirality by Micro-Vortices and Specific Adsorption in Magnetoelectrodeposition. Bull. Chem. Soc. Jpn. 2015, 88, 1479-1485. [CrossRef] 
14. Mogi, I.; Aogaki, R.; Takahashi, K. Fluctuation Effects of Magnetohydrodynamic Micro-Vortices on Odd Chirality in Magnetoelectrolys. Magnetochemistry 2020, 6, 43. [CrossRef]

15. Mogi, I.; Morimoto, R.; Aogaki, R.; Takahashi, K. Surface Chirality in Rotational Magnetoelectrodeposition of Copper Films. Magnetochemistry 2019, 5, 53. [CrossRef]

16. Luo, P.; Zhang, F.; Baldwin, R.P. Constant Potential Amperometric Detection of Underivatized Amino Acids and Peptides at A Copper Electrode. Anal. Chem. 1991, 63, 1702-1707. [CrossRef]

17. Mogi, I.; Aogaki, R.; Takahashi, K. Chiral Surface Formation in Magnetoelectrolysis on Micro-Electrodes. Magnetohydrodynamics 2017, 53, 321-328. [CrossRef]

18. Wachterchauser, G. Before Enzyme and Templates: Theory of Surface Metabolism. Microbiol. Rev. 1988, 52, 452-484. [CrossRef] [PubMed] 